\title{
TIP family aquaporins play role in chloroplast osmoregulation and
} photosynthesis

\author{
Azeez Beebo ${ }^{1 \pi}$, Ahmad Zia ${ }^{2 \pi}$, Christopher R. Kinzel ${ }^{2}$, Andrei Herdean ${ }^{1 \pi}$, Karim \\ Bouhidel $^{3}$, Helmut Kirchhoff ${ }^{2}$, Benoît Schoefs $^{4}$, and Cornelia Spetea ${ }^{1^{*}}$
}

${ }^{1}$ Department of Biological and Environmental Sciences, University of Gothenburg, Box 461, 40530 Gothenburg, Sweden,

${ }^{2}$ Institute of Biological Chemistry, Washington State University, Pullman, WA, USA,

${ }^{3}$ Université de Bourgogne, UMR1347 Agroécologie, ERL CNRS 6300, BP 86510, F-21065 Dijon Cedex, France,

${ }^{4}$ MicroMar, Mer, Molécules, Santé EA2160, LUNAM Université, IUML - FR 3473 CNRS, University of Le Mans, 72085 Le Mans Cedex 9, France

II Present address

Azeez Beebo: TATAA Biocenter, 41103 Gothenburg, Sweden,

Ahmad Zia: Basic Science Unit, University of Hafr Al-Batin, 31991 Hafr Al-Batin, Saudi Arabia,

Andrei Herdean: Climate Change Cluster, University of Technology Sydney, NSW 2007 Australia.

*To whom correspondence should be addressed: Cornelia Spetea, Department of Biological and Environmental Sciences, University of Gothenburg, Box 461, 40530 Gothenburg, Sweden; Tel: +46-317869332; Email: cornelia.spetea.wiklund@bioenv.gu.se

Keywords: chloroplast, aquaporin, tonoplast intrinsic protein, osmoregulation, photosynthesis, Arabidopsis thaliana. 


\section{SUMMARY}

Photosynthetic oxygen evolution by photosystem II requires water supply into the chloroplast to reach the thylakoid lumen. A rapid water flow is also required into the chloroplast for optimal oxygen evolution and to overcome osmotic stress. The mechanisms governing water transport in chloroplasts are largely unexplored. Previous proteomics indicated the presence of three aquaporins from the tonoplast intrinsic protein (TIP) family, TIP1;1, TIP1;2 and TIP2;1, in chloroplast membranes of Arabidopsis thaliana. Here we revisited their location and studied their role in chloroplasts. Localization experiments indicated that TIP2;1 resides in the thylakoid, whereas TIP1;2 is present in both thylakoid and envelope membranes. Mutants lacking TIP1;2 and/or TIP2;1 did not display a macroscopic phenotype when grown under standard conditions. The mutant chloroplasts and thylakoids underwent less volume changes than the corresponding wild type preparations upon osmotic treatment and in the light. Significantly reduced rates of photosynthetic electron transport were obtained in the mutant leaves, with implications on the $\mathrm{CO}_{2}$ fixation rates. However, electron transport rates did not significantly differ between mutants and wild type when isolated thylakoids were examined. Less acidification of the thylakoid lumen was measured in mutants thylakoids, resulting in a slower induction of delta pH-dependent photoprotective mechanisms. These results identify TIP1;2 and TIP2;1 as chloroplast proteins and highlight their importance for osmoregulation and optimal photosynthesis. A third aquaporin, TIP1;1, is present in the chloroplast envelope, and may play role in photosynthesis under excessive light conditions, as based on the weak photosynthetic phenotype of its mutant. 


\section{INTRODUCTION}

Oxygenic photosynthetic organisms including plants, utilize water to produce $\mathrm{O}_{2}$ through the activity of the water-oxidizing complex (WOC) associated with the photosystem II (PSII) complex. PSII as well as PSI, cytochrome $b_{6} f\left(\mathrm{Cytb}_{6} \mathrm{f}\right)$, and the ATP synthase are located in the thylakoid membrane within the chloroplast. Since WOC is bound to the lumenal side of PSII, water must be replenished into the thylakoid lumen at a high flux to sustain its activity. Previously, it has been reported that photosynthetic water oxidation is influenced by changes in chloroplast volume, and that the chloroplast stroma adjusts volume in response to osmotic stress (Robinson 1985; McCain 1995). In addition, it has been shown that the thylakoid lumen also adjusts volume in the light and in response to osmotic stress (Cruz et al., 2001; Kirchhoff et al., 2011). Thus, water oxidation and volume changes in the chloroplast require water movement across envelope and thylakoid membranes.

Water is transported across cell membranes either by free diffusion or by facilitated diffusion through water channels (aquaporins). Aquaporins belong to the major intrinsic protein (MIP) superfamily, which in the model plant Arabidopsis thaliana (Arabidopsis) has 35 members (Johanson et al., 2001). Among Arabidopsis MIPs, there are 13 plasma membrane intrinsic proteins (PIPs) and 10 tonoplast intrinsic proteins (TIPs). MIPs and especially TIPs mediate not only the transport of water but also of other small uncharged solutes such as $\mathrm{H}_{2} \mathrm{O}_{2}$, urea, ammonia, and glycerol, and play role in plant development, growth and tolerance to stress (examples of reviews (Gomes et al., 2009; Li et al., 2013)).

How water molecules enter the chloroplast and reach the thylakoid lumen is largely unexplored. Theoretical calculations indicated low values for the basal water permeability of plant chloroplast envelope and thylakoid membranes, comparable to those of artificial lipid bilayers ((Beebo et al., 2013) and refs. therein). In the same paper, it has been hypothesized that simple non-regulated diffusion may sustain water supply at steady state photosynthetic rates. However, to explain the above-described fast osmoregulatory changes in the chloroplast, an increase in water permeability with the help of aquaporins has been hypothesized. Indeed, the fast water exchange and the low activation energy for water 
transport reported in the literature argue for potential presence of aquaporins in the chloroplast ((Beebo et al., 2013) and refs. therein).

TIP1;1, TIP1;2 and TIP2;1 have previously been localized to the tonoplast in Arabidopsis roots and leaves using immunoblotting and fluorescence microscopy (Hofte et al., 1992; Daniels et al., 1996; Hunter et al., 2007; Beebo et al., 2009; Gattolin et al., 2009). Large-scale analyses of the chloroplast proteome of Arabidopsis did not find any chloroplast-specific aquaporins. They found instead the three TIPs, but their presence was regarded as a probable tonoplast contamination (Ferro et al., 2003; Kleffmann et al., 2004). Later on, TIP2;1 was found by proteomics in thylakoid preparations, whereas TIP1;1 was found in the envelope, and TIP1;2 in both types of preparations (Zybailov et al., 2008; Ferro et al., 2010). Most recent proteomic reports of subfractionated thylakoids found TIP2;1 in the stroma thylakoids, but did not find TIP1;2 in any thylakoid subfractions (Tomizioli et al., 2014).

Altogether, the presence of aquaporins remained unclear, and it was necessary to revisit the occurrence of the three TIP family aquaporins in Arabidopsis chloroplast membranes by direct visualization of fluorescent-tagged TIPs and western blotting. To study their importance for the chloroplast, we have investigated whether mutants lacking either of these proteins display altered osmoregulation and/or photosynthetic activity in standard and high light (HL) conditions.

\section{RESULTS}

\section{Revisiting TIPs subcellular location with emphasis on the chloroplast}

To test whether the three TIP family aquaporins (TIP1;1, TIP1;2 and TIP2;1) are true chloroplast proteins, we have performed a careful investigation of their location using fluorescence microscopy and western blotting with specific antibodies. The GFP gene was fused immediately downstream of the full-length TIP cDNAs and transformed into Arabidopsis plants under the control of each respective endogenous TIP gene promoter (TIPG plants). Using confocal laser-scanning microscopy, the tonoplast was found clearly labelled with green fluorescence in the TIPG leaves (Figure S1), confirming the previously 
reported location for the three TIP isoforms (Beebo et al., 2009; Gattolin et al., 2010). Chloroplasts were not visibly labelled with GFP, probably because chlorophyll (Chl) red autofluorescence was masking GFP fluorescence at the given magnification in intact leaves (Figure S1).

Western blots with anti-GFP antibodies indicated the presence of GFP fusion products in chloroplasts purified by Percoll-gradient from TIPG plants, whereas no crossreaction was observed in chloroplasts from non-transformed Col plants (Figure 1a). Western blots with an anti-V-ATPase antibody verified that the analysed chloroplast preparations were free of vacuolar membranes. These observations determined us to analyse these preparations by fluorescence microscopy. TIPG chloroplasts displayed green fluorescence in a punctate pattern (Figure 1b). The signal was strongest for TIP2;1-GFP and TIP1;2-GFP plants, and weak for the TIP1;1-GFP plants. The even weaker signal in the chloroplasts from non-transformed (Col) plants is most likely autofluorescence from phenolic compounds exhibiting a large emission the 500-600-nm range (Galvez et al., 1998; Brillouet et al., 2013). The difference in signal intensity of the various GFP-TIPs detected in western blot analysis and microscopy is in agreement with the native expression levels of these proteins in the chloroplasts of transgenic lines, as based on the use of native promoters for each construct (see Experimental Procedures).

Western blot analysis using an antibody against a common peptide for TIP1;1 and TIP1;2 indicated a double polypeptide band in leaf extracts from Col plants (Figure 1c). Purified chloroplasts and also thylakoids displayed the upper polypeptide band. The corresponding band was strongest in the envelope fraction. The lower band of the doublet from leaf extracts was only weakly detected in envelope preparations. The identity of the two bands was resolved by western blotting of leaf extracts from mutants lacking either of the two TIPs, as follows: TIP1;2 as the upper and TIP1;1 as the lower band (Figure 2c). Western blots with an anti-TIP2;1 antibody revealed a single polypeptide band in leaf extracts, also detected in purified chloroplasts and thylakoids. A corresponding band was absent in the envelope. Western blots with antibodies for marker proteins of the tonoplast (V-ATPase), plasma membrane $\left(\mathrm{H}^{+}\right.$-ATPase), inner envelope (Tic40) and the thylakoid membrane (Lhcb2) verified the purity of the tested chloroplast membrane preparations 
(Figure 1c). Taken together, these results indicate a dual location of the three TIPs, namely in the vacuole and the chloroplast. Within the chloroplast, TIP1;1 and TIP1;2 are present in the envelope, and TIP1;2 and TIP2;1 in thylakoids. TIP1;1 is a well-known tonoplast marker due to its abundance in this membrane (Beebo et al., 2009; Gattolin et al., 2010), but the fact that the analysed chloroplast preparations were free of tonoplast support a chloroplast location for this TIP as well.

\section{Growth phenotype of the tip mutants}

To investigate the physiological role of the three chloroplast-located TIPs in Arabidopsis, single mutants (tip1;1, tip1;2 and tip2;1) and a double mutant (tip1;2/tip2;1) carrying either T-DNA or a defective Suppressor-mutator (dSpm) transposable element in the corresponding TIP genes (Figure 2a) have been employed. The single tip1;1 mutant generated in a different background (Ws) than the rest of the lines (Col), was previously described by Beebo et al., (2009). Quantitative real-time PCR showed that TIP1;2 and TIP2; 1 mRNA levels were reduced to $<3 \%$ in the respective mutants as compared to Col (Figure 2b). Western blots with TIP1;1/TIP1;2 and TIP2;1 antibodies of leaf extracts indicated the absence of the corresponding protein in the respective tip mutant (Figure 2c). The lack of a macroscopic phenotype has previously been reported for the tipl;1 mutant grown in standard laboratory conditions (Beebo et al., 2009). In our study, no differences were observed at an age of 8 weeks in the appearance of the mutants as compared to plants in the respective wild-type background (Figures $2 \mathrm{~d}$ and S2). Also, no significant differences were obtained in the leaf Chl content, in either the shoot fresh- or dry weight throughout the growth period (Figures 2, e-f and S2, b-c).

\section{Osmoregulatory volume changes in isolated chloroplasts and thylakoids}

We have investigated the effect on osmoregulation of absence of chloroplast TIPs by studying Percoll gradient-purified chloroplasts subjected to osmotic stress for 5 min using a light microscope. When suspended in $0.3 \mathrm{M}$ sucrose (control) buffer, chloroplasts of Col and mutants had an ellipsoid shape and were similar in size (Figure 3). When suspended in water, Col and also tip2; 1 chloroplasts became swollen with irregular shape, whereas those 
of tip1;2 and tip1;2/tip2;1 mutants preserved the ellipsoid shape and size. When incubated in hyper-osmotic solutions containing either ionic $(\mathrm{NaCl})$ or non-ionic (mannitol) osmolyte, all types of chloroplasts decreased in size. Nevertheless, Col and tip2;1 chloroplasts displayed a much more irregular shape, whereas the tip1;2 and tip1;2/tip2;1 mutant chloroplasts were clearly more stable to these osmotic treatments. The lack of osmoregulatory effects in the tip2;1 chloroplasts is in line with a strict thylakoid location for TIP2;1 (Figure 1c). Thus, the reduced volume changes in the tip1;2/tip2;1 chloroplasts are most likely due to the absence of TIP1;2 in the envelope. Even though TIP1;1 resides in the envelope, the chloroplasts isolated from the tip1;1 mutant behaved similarly to those isolated from Ws in all three studied treatments (Figure S3). Taken together, these results strongly indicate that TIP1;2 plays a major role in chloroplast osmoregulation.

Next, we have studied if the lack of TIP1;2 and/or TIP2;1 proteins in Col alters the thylakoid lumen volume changes using $90^{\circ}$ light-scattering measurements at $550 \mathrm{~nm}$ $\left(\Delta \mathrm{A}_{550}\right.$, Figures 4 and $\left.\mathrm{S} 4\right)$. The expansion of the thylakoid lumen in illuminated thylakoids was about $30 \%$ smaller (Figure 4 bar plot) in the mutants as compared to Col. Kinetic analysis of light-induced $\Delta \mathrm{A}_{550}$ revealed that the lower amplitudes in the mutants were caused by about $50 \%$ slower formation of the scattering change (Figure 4 insert). Next, isolated thylakoids were incubated in sugar-free buffer leading to a swollen state. Starting from this state, addition of sorbitol (hyperosmotic agent) results in water efflux and shrinkage of the thylakoid lumen, as demonstrated previously by electron microscopy (Kirchhoff et al., 2011). In Figure 4, the $\Delta \mathrm{A}_{550}$ values at $300 \mathrm{mM}$ sorbitol were set to zero because this concentration corresponds to the osmolarity of the chloroplast stroma. The sorbitol concentration response curves for all three mutants indicated less osmotic shrinkage of the thylakoid lumen than in Col. In other words, higher concentration of sorbitol is required in the mutants to induce similar osmotic shrinkage as in Col thylakoids.

\section{Photosynthetic activity in intact leaves and isolated thylakoids}

The effect on photosynthetic activity of absence of chloroplast-located TIPs in Arabidopsis was studied in terms of $\mathrm{O}_{2}$ evolution, linear electron transport (ETR) and net $\mathrm{CO}_{2}$ assimilation $\left(A_{\mathrm{n}}\right)$ rates in leaves. Light response curves indicated slight but not significant 
decrease of $\mathrm{O}_{2}$ evolution rate at low-intensity of the photosynthetically active radiation (PAR) in the tip1;2, tip2;1 and tip1;2/tip2;1 mutants as compared to Col (Figure 5a left panel). At moderate to high PAR intensities $\left(250-2000 \mu \mathrm{mol} \mathrm{m}^{-2} \mathrm{~s}^{-1}\right)$, the decrease in $\mathrm{O}_{2}$ evolution was significantly more pronounced (by up to 30\%) in the tip2;1 and tip1;2/tip2;1 mutants, indicating the lack of TIP2;1 as the main cause of these effects. Maximal $\mathrm{O}_{2}$ evolution was significantly lower in the tip1;2 and tip2;1 mutants than in Col, and an additive effect was observed in the tip1;2/tip2;1 mutant (Figure 5a right panel). Similar pattern to the $\mathrm{O}_{2}$ evolution was observed for the light response curves of ETR (Figure 5b). The curves for $A_{n}$ versus leaf intercellular $\mathrm{CO}_{2}$ concentration $\left(C_{i}\right)$ showed significantly lower assimilation rates in the mutants than in Col at high concentrations (Figure 5c), most likely due to limitation by the reduced ETR rates (Farquhar et al., 1980).

Non-significant effects on various photosynthetic parameters were observed in the tip1;1 mutant as compared to the Ws background (Figure S5, a-c). Remarkably, steadystate $\mathrm{O}_{2}$ evolution in the tip1;1 mutant became 20\% significantly lower than in Ws leaves

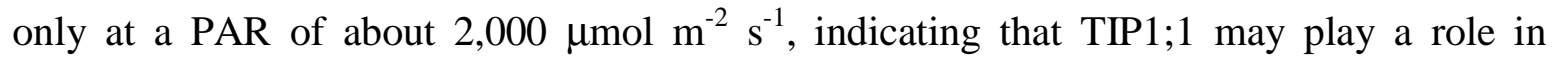
chloroplast photosynthesis only under excessive light conditions. The maximum $\mathrm{O}_{2}$ evolution in the tip1;1 mutant was significantly lower than in Ws leaves (Figure S5a).

Similar abundance of the Lhcb2, D1, AtpB, PsaB, Cytf and RbcL proteins, as determined in western blots with specific antibodies, indicated that the levels of thylakoid photosynthetic complexes and Rubisco were not affected in the mutants as compared to the wild type (Figure S6), and thus cannot explain the observed differences in photosynthetic $\mathrm{O}_{2}$ evolution, ETR and $\mathrm{CO}_{2}$ fixation rates. Taken together, these results reveal that among the studied mutants, tip2;1, tip1;2 and tip1;2/tip2;1 were the most affected in photosynthesis due to the absence of TIP2;1 and TIP1;2 in thylakoids (Figure 2c).

Although mildly, a significantly more pronounced reduction in the maximal photochemical quantum efficiency of PSII (expressed as the Chl fluorescence parameter $\left.F_{\sqrt{ }} / F_{m}\right)$ was observed for the tip2;1 mutant as compared to $\mathrm{Col}$ during $3 \mathrm{~h}$ of illumination at a PAR of $600 \mu \mathrm{mol} \mathrm{m} \mathrm{m}^{-2}$ (HL) (Figure 5d). This indicates an enhanced light sensitivity of the tip2;1 mutant. However, after 3 days of HL treatment, the tip2;1 mutant displayed similar $F_{\downarrow} / F_{m}$ as Col. The other mutants were slightly but not significantly more affected 
than Col throughout the treatment (Figures 5d and S5d). Kinetics of non-photochemical quenching $(N P Q)$ formation showed a significantly lower amplitude of the initial rapid phase in the tip2;1 and tip1;2/tip2;1 mutants, followed by the tip1;2 mutant (Figure 5e), which could be attributed to a lower accumulation of $\mathrm{H}^{+}$in the thylakoid lumen as compared to Col (see below). The tipl;1 mutant displayed only weak but not significant differences from Ws in the $N P Q$ kinetics (Figure S5e).

A consequence of reduced PSII and ETR activity in the mutant leaves is that the light-induced $\mathrm{H}^{+}$pumping is reduced, leading to lower proton-motive force (pmf) across the thylakoid membrane. This is indicated by the significantly lower electrochromic shift $\left(\mathrm{ECS}_{\text {total }}\right)$ amplitude in leaf measurements (Figure 5f), and supported by $\Delta \mathrm{pH}$ measurements with 9-aminoacridine (9-AA) in isolated thylakoid membranes (Figure 5g). Taken together, these data indicate that in the light PSII is not fully operational in the tip 1;2, tip2;1 and tip 1;2/tip2;1 mutants, leading to increased photosensitivity and reduced photoprotection.

To study reasons for reduced PSII activity in these mutants, we investigated the photosynthetic reactions in more detail using either intact leaves or isolated thylakoids. Quantification of electron transport components by difference absorption spectroscopy revealed no significant differences in the levels of PSII, Cytb ${ }_{6} \mathrm{f}$ and PSI complexes between the mutants and Col leaves (Table S1), in accordance with the western blot data (Figure S6). The high-potential chain of electron transport system (between Cytf and $\mathrm{P}_{700}$ ) was probed by analysing relaxation kinetics after a saturating 200-ms light pulse in intact leaves (Kirchhoff et al., 2011). At the end of the light pulse, the plastoquinone pool was fully reduced and electron flow into the oxidized high-potential chain was monitored by $\mathrm{Cytf}^{+}$ and $\mathrm{P}_{700}{ }^{+}$dark relaxation kinetics. Both the unchanged halftime of $\mathrm{P}_{700}{ }^{+}$relaxation (Table 1) as well as the redox equilibration between $\mathrm{Cytf}^{+}$and $\mathrm{P}_{700}{ }^{+}$(Figure S7) indicated that electron flow in the high-potential chain was unaffected in the three mutants. The $F_{\mathrm{v}} / F_{\mathrm{m}}$ parameter was indistinguishably high between the mutants and Col leaves (Table 1), indicating that in dark-adapted plants PSII is fully operational upon application of a strong saturation pulse. Notably, the $\Phi_{\text {II }}$ parameter was significantly lower in the mutant leaves (Table 1), consistent with the light response ETR curves (Figure 5b), indicating that in continuous light PSII does not function properly. 
When using thylakoids isolated from Arabidopsis Col and mutant leaves, no significant differences were observed for the light-saturated PSII $\mathrm{O}_{2}$ evolution rate probed with the electron acceptor dimethyl-benzoquinone in the presence of the uncoupler nigericin (PSII, Table 1). This is in disagreement with the data from leaf measurements (Figure 5a). Furthermore, $\mathrm{O}_{2}$ evolution in the presence of methyl viologen (MV, electron acceptor from PSI) and of nigericin revealed no significant reduction for the mutants as compared to Col (ETR, Table 1). Cytb ${ }_{6} \mathrm{f}$ activity was also indistinguishable between Col and mutants $\left(\mathrm{Cytb}_{6} \mathrm{f}\right.$, Table 1$)$. Taken together, these data indicate that water availability is not limiting for the full operational of PSII in thylakoids isolated from the tip mutants. Therefore, other factors than water deficiency in the thylakoid lumen may explain the reduced PSII activity in the mutant leaves.

To further explore the role of TIP2;1 in photosynthetic regulation, we performed experiments under fluctuating light. In transition from low to high light, the tip2;1 mutant displayed slower NPQ induction but without changing the PSII quantum yield (Figure S8). In the TIP2;1-GFP transgenic plants, NPQ was induced faster, resulting in less electron transport through PSII than in Col. These data indicate a role for TIP2;1 in fluctuating light conditions where relatively fast changes in $\Delta \mathrm{pH}$ across the thylakoid membrane are required. Furthermore, ammonia known to regulate water uptake and aquaporins (Wang et al. 2016) appears to enhance the effects seen in the mutants (Figure S9).

\section{DISCUSSION}

The molecular mechanisms governing water transport into and within the chloroplast are largely unexplored. Nevertheless, recent theoretical considerations based on published experimental data suggested the presence of aquaporins in thylakoids (Beebo et al., 2013). In this study, we bring experimental proof for the occurrence of three TIP family aquaporins (TIP1;1, TIP1;2 and TIP2;1) in Arabidopsis chloroplast membranes, and also bring insights into their role in chloroplasts. We propose that TIP1;2 and TIP2;1 are housekeeping chloroplast aquaporins, which importantly contribute to its water balance and 
photosynthesis, whereas TIP1;1 may contribute to the efficiency of photosynthesis only in acute light stress.

\section{TIP1;1, TIP1;2 and TIP2;1 have dual location to the vacuole and the chloroplast}

TIPs are located mainly in the tonoplast, and some of them (e.g., TIP1;1) have been extensively used as tonoplast markers (Beebo et al., 2009; Gattolin et al., 2010). Since they do not possess targeting sequences, other subcellular locations could not be ruled out. Proteomics of chloroplast preparations gave contradictory results about the three TIP isoforms (Ferro et al., 2010; Tomizioli et al., 2014). Therefore, individual localization studies were requested. In this work, green fluorescence microscopy revealed that, in addition to the vacuole, Arabidopsis TIP1;1, TIP1;2 and TIP2;1 were associated with the chloroplast as punctate structures (Figures $1 \mathrm{~b}$ and S1). The fact that in the TIP1;1-GFP line, the GFP signal was detected primarily in a few punctate structures near the organelle periphery (Figure 1a) is in line with its low expression level in chloroplasts, as detected by western blotting (Figure 1, a and c). Similar punctate fluorescence pattern has been reported for other chloroplast proteins fused to GFP, namely dynamin FZL, GTPase ObgC, glutamine synthase GLN2, and mechanosensitive channel MSCL1 (Taira et al., 2004; Gao et al., 2006; Nakayama et al., 2007; Bang et al., 2009). In the case of ObgC, this pattern was explained by a dimerization-type of protein-protein interaction (Bang et al., 2009). Using bimolecular fluorescence complementation in yeast, Murozuka et al., (2013) have shown strong interactions between TIP1;2 and TIP2;1, and proposed a role in targeting and/or regulation. FZL was found dually located to the envelope and the thylakoid membrane (Gao et al., 2006), GLN2 was found in both the chloroplast stroma and mitochondrial matrix (Taira et al., 2004), and MSCL1 was found in the cytoplasm and the chloroplast (Nakayama et al., 2007). Thus, the punctate pattern indicates location in distinct areas in the membrane and it may require interaction between TIPs and/or to dual targeting to the vacuole and the chloroplast. Other examples of dually located aquaporins are TIP3;1 and TIP3;2, which were found in the plasma membrane and the tonoplast (Gattolin et al., 2011). AQP1 was localized to the plasma membrane and also to the chloroplast envelope in Nicotiana tabacum (Uehlein et al., 2003; Uehlein et al., 2008). Interestingly, the substrate 
specificity was found to be location-dependent since only the plasma membrane AQP1 transported water, whereas the envelope AQP1 facilitated diffusion of $\mathrm{CO}_{2}$. Whether the type of substrate is location-dependent in the case of the three TIPs needs further investigations.

\section{Chloroplast-located TIPs are important for chloroplast osmoregulation and optimal photosynthesis}

Although they may co-exist on the same tonoplast, transcriptomics data and fluorescence microscopy of fusion proteins indicated distinct tissue and organ expression pattern for the three TIPs in Arabidopsis (Schmid et al., 2005; Hunter et al., 2007; Gattolin et al., 2009). TIP1;1 is the most widespread TIP isoform in Arabidopsis, being present in both roots and leaves (Hunter et al., 2007; Beebo et al., 2009; Gattolin et al., 2009). TIP1;2 and TIP2;1 are mostly expressed in leaves and have only a limited expression in roots (Hunter et al., 2007; Gattolin et al., 2009). Arabidopsis tip1;1 and tip1;2 mutants did not show any macroscopic phenotype in previous reports (Schussler et al., 2008; Beebo et al., 2009). In this study, in addition to these mutants, tip2;1 and also tip1;2/tip2;1 did not display any visual phenotype when grown under standard conditions (Figure 2 and S2). Our and previous observations (Beebo et al., 2009) that inactivation of one or another TIP has little or no effect on plant growth under standard conditions indicate redundant functions among this type of aquaporins.

The most beneficial action of aquaporins is to provide massive water flow in response to osmotic stress, leading to rapid changes in cell volume. Indeed, volume changes of the thylakoid lumen and chloroplast stroma have been previously reported upon osmotic shock and also in the light (Robinson 1985; McCain 1995; Cruz et al., 2001; Kirchhoff et al., 2011). In this study, western blotting using anti-TIP antibodies indicated an envelope location for TIP1;1, a thylakoid location for TIP2;1 and a dual envelope/thylakoid location for TIP1;2 (Figure 1c). Our observations of inhibited osmoregulatory volume changes in the chloroplasts of tip1;2 and tip1;2/tip2;1 mutants (Figure 3) indicate a main role for TIP1;2 protein in mediating water movement across the chloroplast envelope. The observations of reduced thylakoid volume changes in the light and also upon osmotic 
treatment of tip1;2, tip2;1 and tip1;2/tip2;1 mutants (Figure 4) point to a role for TIP1;2 and TIP2;1 in water movement across the thylakoid membrane.

Water is a substrate for photosynthetic $\mathrm{O}_{2}$ evolution inside the thylakoid lumen. Recent calculations based on published data indicated that free diffusion could sustain water oxidation at steady state photosynthetic rates, and that aquaporins may not be absolutely required (Beebo et al., 2013). In this study, we show no difference in photosynthetic $\mathrm{O}_{2}$ evolution between $\mathrm{Col}$ and the tip1;2, tip2;1 and tip1;2/tip2;1 mutants thylakoids (Table 1), consistent with this hypothesis. However, significantly reduced $\mathrm{O}_{2}$ evolution rates were obtained in mutant leaves (Figure 4a). There are several possible explanations for this discrepancy. 1) The lack of tonoplast-located TIPs may indirectly affect photosynthesis in intact leaves, but not in isolated thylakoids, since they are devoid of tonoplast (Figure 1c). However, this possibility is not very likely since absence of the most abundant TIP isoform in the tonoplast (TIP1;1) had only marginal effects on photosynthesis in leaves (Figure S5). 2) In isolated thylakoids, water supply into the lumen via free diffusion may sustain PSII photochemistry, making aquaporins not absolutely required, as proposed by Beebo et al., (2013). However, aquaporin-dependent processes, such as chloroplast osmoregulation, may limit PSII activity in leaves. Osmoregulatory volume changes were observed in chloroplasts isolated from wild type, tip1;1 and tip2;1 but were inhibited in the tip1;2 and tip1;2/tip2;1 mutant chloroplasts. Thus, the possibility that defects in vacuole-located TIPs caused the observed effects is excluded. The inhibited osmoregulatory volume changes of chloroplasts isolated from the mutant leaves may reduce the dynamics in thylakoid organization, hence its optimal function in photosynthesis.

The average pre-exchange lifetime of water in the thylakoid lumen of $10 \mathrm{~ms}$ indicates that the lumen is refilled $3 \times 10^{4}$ fold faster than the photosynthetic water oxidation when expressed per sec (Beebo et al., 2013). Thus, if aquaporins are responsible for this high exchange activity, it is for the optimal function of the thylakoid rather than primarily to sustain water oxidation. This reasoning could explain the mild effects on photosynthetic $\mathrm{O}_{2}$ evolution at moderate to high PAR intensities in the tip1;2, tip2,1 and tip1;2/tip2;1 mutants (Figure 5). The tipl;1 mutant was affected in $\mathrm{O}_{2}$ evolution only at extremely high PAR, 
suggesting a role for TIP1;1 under acute light stress in chloroplasts. Finally, Chl fluorescence measurements indicated an earlier HL-induced inactivation and to a larger extent in the tip2;1 mutant than in Col and the other tip mutants (Figure 5), in line with the thylakoid location of TIP2;1. Although significant differences were observed, the effects were relatively mild. One potential explanation could be redundancy of the three studied TIPs and of other aquaporins in the envelope as detected by mass spectrometry (e.g., PIPs, Ferro et al., (2010)).

\section{CONCLUSIONS}

The finding of a chloroplast location in addition to the vacuolar one for the three TIP family aquaporins allowed us to validate the previous proteomics findings. Phenotypic characterization of their mutants allowed us to understand that TIP1;2 and TIP2;1 are necessary for chloroplast osmoregulation and optimal photosynthesis, whereas TIP1;1 may act in extreme light stress conditions. The two roles may be interrelated by a mechanism that requires further investigations. Other questions that should be the focus of future studies are the signals and pathways of TIPs trafficking to the chloroplast. The mechanisms of TIP trafficking in the cytosol are poorly understood, but it is thought that they are delivered to the vacuole by vesicles from the endoplasmic reticulum via Golgi-dependent and independent pathways (Rivera-Serrano et al., 2012). Few examples of chloroplast proteins have been reported to use Golgi vesicles (Kitajima et al., 2009) and putative components of a vesicular transport system to and within the chloroplast have been predicted (Khan et al., 2013). Additional question to be resolved is whether each of the chloroplast TIP isoforms has a specific role or they just provide sufficient redundancy to ensure that cellular water homeostasis remains under control for optimal chloroplast function.

\section{EXPERIMENTAL PROCEDURES}

\section{Plant material and growth conditions}


Arabidopsis plants (ecotypes $\mathrm{Col}$ and $\mathrm{Ws}$ ), the TIPG transgenic and tip mutants were grown using a $16 \mathrm{~h}$ dark $/ 8 \mathrm{~h}$ light cycle $\left(120 \mu \mathrm{mol}\right.$ photons $\left.\mathrm{m}^{-2} \mathrm{~s}^{-1}\right)$ at $20^{\circ} \mathrm{C}$ and $60 \%$ relative humidity) in a growth chamber (CLF PlantMaster, Plant Climatics GmbH, Wertingen, Germany) for 8 weeks, if not otherwise indicated. The Arabidopsis TIP1;1GFP and the tip1;1 mutant have been previously described (Beebo et al., 2009). The construction of the TIP1;2-GFP and TIP2;1-GFP fusions and Arabidopsis transformation, the tip1;2, tip2;1 and tip1;2/tip2;1 mutants are described in Methods S1, Tables S2 and S3. For localization studies, chloroplasts were isolated and purified on a Percoll gradient according to (Thuswaldner et al., 2007). Following lysis of purified chloroplasts, thylakoids and envelope were purified on a sucrose gradient as described (Thuswaldner et al., 2007). Protein concentration was determined using Bio Rad DC ${ }^{\mathrm{TM}}$ protein Assay (Bio Rad). For activity studies, chloroplasts and thylakoids were prepared by a fast method at $4^{\circ} \mathrm{C}$. In brief, dark-adapted rosette leaves were harvested and homogenized in grinding medium (400 mM sorbitol, $20 \mathrm{mM}$ Tricine, $10 \mathrm{mM}$ EDTA, $10 \mathrm{mM} \mathrm{NaHCO}_{3}$ and $0.15 \% \mathrm{BSA}$ at $\mathrm{pH} \mathrm{8.4).} \mathrm{The}$ homogenate was then filtered, centrifuged at 2,000 x $g$ for $2 \mathrm{~min}$, and the resulting pellet was resuspended in washing medium (400 mM sorbitol, $20 \mathrm{mM}$ HEPES, $2.5 \mathrm{mM}$ EDTA, 5 $\mathrm{mM} \mathrm{MgCl} 2,10 \mathrm{mM} \mathrm{NaHCO}$ and $0.15 \% \mathrm{BSA}_{2}$ at $\mathrm{pH}$ 7.6). Final pellet was obtained by centrifugation at 2,000 $\mathrm{x} g$ for $1 \mathrm{~min}$, and resuspended in the washing medium. Chl concentration was measured in $80 \%$ (w/v) acetone (Porra 2002). Leaf Chl content was measured using a portable device (CCM-200 plus, Optic-science Inc, NL).

\section{Microscopy}

For localization studies, leaf sections of TIPG and Col leaves and Percoll-gradient isolated chloroplasts were mounted in water under a coverslip. Fluorescence detection was conducted with a LSM 700 Axio Observer.Z1 confocal microscope (Carl Zeiss, Germany). Excitation wavelengths and emission filters were $488 \mathrm{~nm} /$ band-pass 506-538 $\mathrm{nm}$ for GFP and $488 \mathrm{~nm} /$ bandpass 664-696 $\mathrm{nm}$ for Chl. Images were processed using Photoshop CS5 software (Adobe Systems, San Jose, CA). For osmotic changes, purified chloroplasts were resuspended in control buffer $(50 \mathrm{mM}$ Tricine $\mathrm{pH} 7.5$ containing $5 \mathrm{mM} \mathrm{MgCl} 2$ and $330 \mathrm{mM}$ sucrose), in water, $0.5 \mathrm{M} \mathrm{NaCl}$ or $1 \mathrm{M}$ mannitol and incubated for $5 \mathrm{~min}$ on ice before 
examination, as described (Zhang et al., 2012). Photos of the chloroplasts were taken with a light microscope in phase contrast mode (Axioplan2 Imaging, Zeiss, Germany).

\section{Light scattering}

Changes in $90^{\circ}$ light scattering were measured with a HoribaYvon Fluoromax 4 spectrofluorometer $\left(\lambda_{\mathrm{ex}}=\lambda_{\mathrm{em}}=550 \mathrm{~nm}\right.$; bandwidth: $\left.2 \mathrm{~nm}\right)$ on isolated thylakoid membranes prepared from dark-adapted plants (Kirchhoff et al., 2011). Membranes (20 $\mu \mathrm{g}$

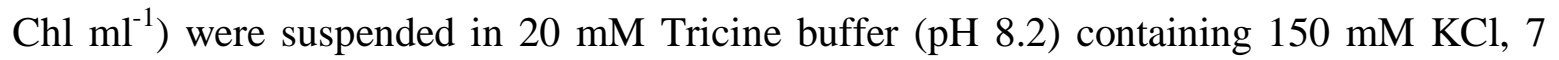
$\mathrm{mM} \mathrm{MgCl}{ }_{2}$ and incubated for 10 min to obtain swollen lumen and subsequently incubated for $5 \mathrm{~min}$ with sorbitol at various concentrations to induce shrinkage before recording the spectra. These incubation times were initially measured and selected to ensure stable absorbance signal by complete mixing of thylakoid suspension and osmoticum for each measurement. Light scattering changes in the presence of $50 \mu \mathrm{M} \mathrm{MV}$ were induced by applying red light $\left(1,000 \mu \mathrm{mol} \mathrm{m} \mathrm{m}^{-2} \mathrm{~s}^{-1}\right.$, Leica KL 1500 LED). Scattering changes were measured first in dark for $100 \mathrm{~s}$, then the thylakoids were light treated for $400 \mathrm{~s}$ followed by dark measurement for 500 s. All light-induced scattering changes were measured with continuous stirring while osmoticum induced changes were measured with stirring switched off 2 min prior to each data recording, to avoid signal noise.

\section{Measurement of photosynthetic activity}

Response curves of $\mathrm{O}_{2}$ evolution rate versus irradiance were recorded in leaf discs using Clark-type electrode (Hansatech, Pentney, King's Lynn, U.K.) at $20^{\circ} \mathrm{C}$. The curves were fitted according to Nguyen-Deroche Tle et al., (2012) using CurveExpert software, to calculate the maximum photosynthetic $\mathrm{O}_{2}$ evolution.

Chl $a$ fluorescence was measured using a Hansatech FMS1 fluorimeter on intact plants at the end of the 16-h dark phase or following illumination for 6 min with actinic light of $675-725 \mu \mathrm{mol}$ photon $\mathrm{m}^{-2} \mathrm{~s}^{-1} . F_{v} / F_{m}$ and $\Phi_{\mathrm{II}}$ were deduced as described (Baker 2008). Light response curves of ETR were determined based on Chl $a$ fluorescence measured on intact plants using Dual-PAM-100 (Walz, Effeltrich, Germany) as described (Lundin et al., 2007). 
$A_{n} / C_{i}$ response curves were recorded using a leaf gas-exchange instrument (LI6400XT, LiCOR, Lincoln, Nebraska, USA) at saturating PAR of $1,800 \mu \mathrm{mol} \mathrm{m}^{-2} \mathrm{~s}^{-1}$ and $25^{\circ} \mathrm{C}$, with stepwise increase of external $\mathrm{CO}_{2}$ concentration (with 3-5 min of adaptation). For determination of $N P Q$, slow kinetics of Chl fluorescence induction were recorded in intact plants, exposed to PAR of $800 \mu \mathrm{mol}$ photon $\mathrm{m}^{-2} \mathrm{~s}^{-1}$, followed by darkness.

Steady-state rates of $\mathrm{O}_{2}$ evolution were also measured in isolated thylakoids using Clark-type electrode at $20^{\circ} \mathrm{C}$. The thylakoids $\left(20 \mu \mathrm{g} \mathrm{Chl} \mathrm{ml}{ }^{-1}\right)$ in $25 \mathrm{mM}$ HEPES buffer (pH 7.5), $300 \mathrm{mM}$ sorbitol, $40 \mathrm{mM} \mathrm{KCl}$ and $7 \mathrm{mM} \mathrm{MgCl}_{2}$ were illuminated with saturating red light $\left(3,000 \mu \mathrm{mol}\right.$ photon $\left.\mathrm{m}^{-2} \mathrm{~s}^{-1}\right)$ in the presence of $1 \mu \mathrm{M}$ nigericin. PSII activity was assayed by using $1.5 \mathrm{mM}$ dimethyl-benozoquinone as electron acceptor. Linear ETR was measured with $100 \mu \mathrm{M}$ MV as the electron acceptor for PSI. MV catalyzes the reduction of $\mathrm{O}_{2}$ by PSII to $\mathrm{H}_{2} \mathrm{O}_{2}$ (Mehler reaction). Overall for each oxygen molecule consumed by Mehler reaction four electrons were transported from water to PSI via the linear electron transport chain $\left(2 \mathrm{H}_{2} \mathrm{O}+2 \mathrm{O}_{2} \Leftrightarrow 2 \mathrm{H}_{2} \mathrm{O}_{2}+\mathrm{O}_{2}\right)$. To avoid cleavage of $\mathrm{H}_{2} \mathrm{O}_{2}$ by catalase that would disturb the strict electron to oxygen stoichiometry, $1 \mathrm{mM}$ sodium azide was added to the reaction cocktail.

The activity of $\mathrm{Cytb}_{6}$ f complex was determined using $25 \mathrm{mM}$ reduced duroquinol $\left(\mathrm{DQH}_{2}\right)$ as the electron donor (replaces plastoquinone) and $100 \mu \mathrm{M} \mathrm{MV}$ as the PSI electron acceptor. This is a measure of linear electron flow at its maximum capacity.

\section{Difference spectroscopy}

The contents of PSII and $\mathrm{Cytb}_{6} \mathrm{f}$ complex were measured in isolated thylakoids incubated in $0.003 \%$ (w/v) $\beta$-dodecyl-maltoside by difference spectroscopic quantifications of $\mathrm{Cytb}_{559}$ and $\mathrm{Cytb}_{6}$, respectively (Kirchhoff et al., 2002). The spectra were recorded with a Hitachi U3900 spectrometer (spectral range, 540-575 nm; 2-nm slit width) and analysed as described (Kirchhoff et al., 2002). Difference absorption spectroscopy of photooxidizable Cytf and $\mathrm{P}_{700}$ in leaves was measured with home-built flash spectrometer (Kirchhoff et al., 2011). For measurements, the leaf material was incubated in $5 \mathrm{mM} \mathrm{MV}$-soaked Kimwipes for $30 \mathrm{~min}$ in darkness. $\mathrm{P}_{700}$ redox signals were derived from absorption changes at 820 and 
900 nm, while Cytf redox kinetics were derived from absorbance changes at 554, 545 and $572 \mathrm{~nm}$ (Kirchhoff et al., 2004).

\section{Measurement of pmf and $\Delta \mathrm{pH}$}

Pmf in leaves was deduced from ECS $_{\text {total }}$ signals at $520 \mathrm{~nm}$ corrected by $550 \mathrm{~nm}$ (Klughammer et al., 2013), measured with a home-built flash spectrometer (Kirchhoff et al., 2011). Pmf was determined at the end of a 10 min illumination period (500 $\mu \mathrm{mol}$ photons $\mathrm{m}^{-2} \mathrm{~s}^{-1}$ ). Light-induced $\Delta \mathrm{pH}$ was measured in freshly isolated thylakoids by 9-AA fluorescence quenching (Schuldinger et al., 1972). The magnitude of the $\Delta \mathrm{pH}$ across thylakoid membranes was calculated from 9-AA quenching as described (Van et al., 1987). Measurements were performed with a HoribaYvon Fluoromax 4 spectrofluorometer $\left(\lambda_{\mathrm{ex}}=\right.$ $400 \mathrm{~nm}$; bandwidth: $2 \mathrm{~nm} ; \lambda_{\mathrm{em}}=455 \mathrm{~nm}$; bandwidth: $\left.5 \mathrm{~nm}\right)$ with thylakoids $(20 \mu \mathrm{g} \mathrm{Chl} \mathrm{ml}$ ${ }^{1}$ ) suspended in measuring buffer containing $6 \mu \mathrm{M} 9-\mathrm{AA}$ and $50 \mu \mathrm{M} \mathrm{MV}$ under continuous stirring. $\Delta \mathrm{pH}$ was induced by red light of $1,000 \mu \mathrm{mol} \mathrm{m} \mathrm{m}^{-2} \mathrm{~s}^{-1} .1 \mu \mathrm{M}$ of nigericin was added after each light cycle to obtain maximum fluorescence value during darkness.

Protein analysis. Electrophoretic separation of proteins was carried out in $12 \%$ (w/v) acrylamide SDS-gels. Following electrotransfer to PVDF membranes, proteins were identified by western blotting. Following antibodies from Agrisera (Vännäs, Sweden) were used: TIP2;1, TIP1;1/TIP1;2, actin, V-ATPase, $\mathrm{H}^{+}$-ATPase, Tic40, D1, Lhcb2 and RbcL. The anti-GFP antibody was obtained from Roche Diagnostics (Indianapolis, IN, USA).

\section{Accession numbers}

Sequence data from this article can be found in the EMBL/Gen-Bank data libraries under accession numbers At2g36830 (TIP1;1), At3g26520 (TIP1;2) and At3g16240 (TIP2;1).

\section{ACKNOWLEDGEMENTS}

We are very grateful to the Centre for Cellular Imaging (Sahlgrenska Academy, Gothenburg University) for the use of confocal microscopes and helpful assistance. This 
work was supported by the Swedish Research Council and the Enkvists Foundation (C.S.). H.K. received support from the National Science Foundation (NSF-MCB115871), the United States-Israel Binational Agricultural Research and Development Fund (BARD US4334-10), the US Department of Agriculture (ARC grant WNP00775), and Washington State University. This work was also supported by the French Ministère de l'Enseignement Supérieur et de la Recherche and INRA (K.B., B.S.) and University of Le Mans (B.S.). Travel Grants were provided by Helge Ax:Son Johnsons Foundation (A.B.), Adlebertska stiftelsen and the University of Le Mans (Grant: Chercheur haut niveau) (C.S.).

\section{SUPPORTING INFORMATION}

Supplemental Methods: Construction of TIP-GFP fusions and screening of tip mutants

\section{Supplemental Tables and Figures}

Table S1. Levels of photosynthetic complexes in Col and tip mutants.

Table S2. Primers for GFP fusions PCR cloning.

Table S3. AGI codes, gene names and primer sequences for quantitative RT-PCR.

Figure S1. Confocal microscopic images of leaves from Col wild type plants and lines expressing TIP-GFP fusion proteins.

Figure S2. Growth of Ws plants and the tip1;1 mutant.

Figure S3. Osmoregulatory volume changes in Ws and tip1;1 mutant.

Figure S4. Light and osmoticum induced changes in isolated thylakoids.

Figure S5. Photosynthetic activity in the tip1;1 mutant and Ws plants.

Figure S6. Western blot analysis of marker proteins for photosynthetic complexes.

Figure S7. Effect on intersystem transport and PSI.

Figure S8. Dynamics of photosynthesis and photoprotection in fluctuating light.

Figure S9. The effect of $\left(\mathrm{NH}_{4}\right)\left(\mathrm{NO}_{3}\right)$ on the dynamics of photosynthesis and photoprotection in fluctuating light.

\section{REFERENCES}


Baker, N.R. (2008) Chlorophyll fluorescence: a probe of photosynthesis in vivo. Annu Rev Plant Biol, 59, 89-113.

Bang, W.Y., Hata, A., Jeong, I.S., Umeda, T., Masuda, T., Chen, J., Yoko, I., Suwastika, I.N., Kim, D.W., Im, C.H., Lee, B.H., Lee, Y., Lee, K.W., Shiina, T. and Bahk, J.D. (2009) AtObgC, a plant ortholog of bacterial Obg, is a chloroplasttargeting GTPase essential for early embryogenesis. Plant Mol. Biol., 71, 379-390.

Beebo, A., Mathai, J.C., Schoefs, B. and Spetea, C. (2013) Assessment of the requirement for aquaporins in the thylakoid membrane of plant chloroplasts to sustain photosynthetic water oxidation. FEBS Lett., 587, 2083-2089.

Beebo, A., Thomas, D., Der, C., Sanchez, L., Leborgne-Castel, N., Marty, F., Schoefs, B. and Bouhidel, K. (2009) Life with and without AtTIP1;1, an Arabidopsis aquaporin preferentially localized in the apposing tonoplasts of adjacent vacuoles. Plant Mol. Biol., 70, 193-209.

Brillouet, J.M., Romieu, C., Schoefs, B., Solymosi, K., Cheynier, V., Fulcrand, H., Verdeil, J.L. and Conejero, G. (2013) The tannosome is an organelle forming condensed tannins in the chlorophyllous organs of Tracheophyta. Ann. Bot., 112, 1003-1014.

Cruz, J.A., Salbilla, B.A., Kanazawa, A. and Kramer, D.M. (2001) Inhibition of plastocyanin to $\mathrm{P}(700)(+)$ electron transfer in Chlamydomonas reinhardtii by hyperosmotic stress. Plant Physiol., 127, 1167-1179.

Daniels, M.J., Chaumont, F., Mirkov, T.E. and Chrispeels, M.J. (1996) Characterization of a new vacuolar membrane aquaporin sensitive to mercury at a unique site. Plant Cell, 8, 587-599.

Farquhar, G.D., Caemmerer, S. and Berry, J.A. (1980) A biochemical model of photosynthetic CO2 assimilation in leaves of C3 species. . Planta, 149, 78-90.

Ferro, M., Brugiere, S., Salvi, D., Seigneurin-Berny, D., Court, M., Moyet, L., Ramus, C., Miras, S., Mellal, M., Le Gall, S., Kieffer-Jaquinod, S., Bruley, C., Garin, J., Joyard, J., Masselon, C. and Rolland, N. (2010) AT_CHLORO, a 
comprehensive chloroplast proteome database with subplastidial localization and curated information on envelope proteins. Mol Cell Proteomics, 9, 1063-1084.

Ferro, M., Salvi, D., Brugiere, S., Miras, S., Kowalski, S., Louwagie, M., Garin, J., Joyard, J. and Rolland, N. (2003) Proteomics of the chloroplast envelope membranes from Arabidopsis thaliana. Mol Cell Proteomics, 2, 325-345.

Galvez, S., Roche, O., Bismuth, E., Brown, S., Gadal, P. and Hodges, M. (1998) Mitochondrial localization of a NADP-dependent [corrected] isocitrate dehydrogenase isoenzyme by using the green fluorescent protein as a marker. Proc. Natl. Acad. Sci. USA, 95, 7813-7818.

Gao, H., Sage, T.L. and Osteryoung, K.W. (2006) FZL, an FZO-like protein in plants, is a determinant of thylakoid and chloroplast morphology. Proc. Natl. Acad. Sci. USA, 103, 6759-6764.

Gattolin, S., Sorieul, M. and Frigerio, L. (2010) Tonoplast intrinsic proteins and vacuolar identity. Biochem. Soc. Trans., 38, 769-773.

Gattolin, S., Sorieul, M. and Frigerio, L. (2011) Mapping of tonoplast intrinsic proteins in maturing and germinating Arabidopsis seeds reveals dual localization of embryonic TIPs to the tonoplast and plasma membrane. Mol Plant, 4, 180-189.

Gattolin, S., Sorieul, M., Hunter, P.R., Khonsari, R.H. and Frigerio, L. (2009) In vivo imaging of the tonoplast intrinsic protein family in Arabidopsis roots. BMC Plant Biol., 9, 133.

Gomes, D., Agasse, A., Thiebaud, P., Delrot, S., Geros, H. and Chaumont, F. (2009) Aquaporins are multifunctional water and solute transporters highly divergent in living organisms. Biochim. Biophys. Acta, 1788, 1213-1228.

Hofte, H., Hubbard, L., Reizer, J., Ludevid, D., Herman, E.M. and Chrispeels, M.J. (1992) Vegetative and seed-specific forms of tonoplast intrinsic protein in the vacuolar membrane of Arabidopsis thaliana. Plant Physiol., 99, 561-570.

Hunter, P.R., Craddock, C.P., Di Benedetto, S., Roberts, L.M. and Frigerio, L. (2007) Fluorescent reporter proteins for the tonoplast and the vacuolar lumen identify a single vacuolar compartment in Arabidopsis cells. Plant Physiol., 145, 1371-1382. 
Johanson, U., Karlsson, M., Johansson, I., Gustavsson, S., Sjövall, S., Fraysse, L., Weig, A.R. and Kjellbom, P. (2001) The complete set of genes encoding major intrinsic proteins in arabidopsis provides a framework for a new nomenclature for major intrinsic proteins in plants. Plant Physiol., 126, 1358-1369.

Khan, N.Z., Lindquist, E. and Aronsson, H. (2013) New putative chloroplast vesicle transport components and cargo proteins revealed using a bioinformatics approach: an Arabidopsis model. PLoS One, 8, e59898.

Kirchhoff, H., Hall, C., Wood, M., Herbstova, M., Tsabari, O., Nevo, R., Charuvi, D., Shimoni, E. and Reich, Z. (2011) Dynamic control of protein diffusion within the granal thylakoid lumen. Proc. Natl. Acad. Sci. USA, 108, 20248-20253.

Kirchhoff, H., Mukherjee, U. and Galla, H.J. (2002) Molecular architecture of the thylakoid membrane: lipid diffusion space for plastoquinone. Biochemistry (Mosc). 41, 4872-4882.

Kirchhoff, H., Tremmel, I., Haase, W. and Kubitscheck, U. (2004) Supramolecular photosystem II organization in grana thylakoid membranes: evidence for a structured arrangement. Biochemistry (Mosc). 43, 9204-9213.

Kitajima, A., Asatsuma, S., Okada, H., Hamada, Y., Kaneko, K., Nanjo, Y., Kawagoe, Y., Toyooka, K., Matsuoka, K., Takeuchi, M., Nakano, A. and Mitsui, T. (2009) The rice alpha-amylase glycoprotein is targeted from the Golgi apparatus through the secretory pathway to the plastids. Plant Cell, 21, 2844-2858.

Kleffmann, T., Russenberger, D., von Zychlinski, A., Christopher, W., Sjolander, K., Gruissem, W. and Baginsky, S. (2004) The Arabidopsis thaliana chloroplast proteome reveals pathway abundance and novel protein functions. Curr. Biol., 14, 354-362.

Klughammer, C., Siebke, K. and Schreiber, U. (2013) Continuous ECS-indicated recording of the proton-motive charge flux in leaves. Photosynth Res, 117, 471-487.

Li, G., Santoni, V. and Maurel, C. (2013) Plant aquaporins: roles in plant physiology. Biochim. Biophys. Acta, 1840, 1574-1582. 
Lundin, B., Hansson, M., Schoefs, B., Vener, A.V. and Spetea, C. (2007) The Arabidopsis $\mathrm{PsbO} 2$ protein regulates dephosphorylation and turnover of the photosystem II reaction centre D1 protein. Plant J., 49, 528-539.

McCain, D.C. (1995) Combined effects of light and water stress on chloroplast volume regulation. Biophys. J., 69, 1105-1110.

Murozuka, E., Hanisch, S., Pomorski, T.G., Jahn, T.P. and Schjoerring, J.K. (2013) Bimolecular fluorescence complementation and interaction of various Arabidopsis major intrinsic proteins expressed in yeast. Physiol. Plant., 148, 422-431.

Nakayama, Y., Fujiu, K., Sokabe, M. and Yoshimura, K. (2007) Molecular and electrophysiological characterization of a mechanosensitive channel expressed in the chloroplasts of Chlamydomonas. Proc. Natl. Acad. Sci. USA, 104, 5883-5888.

Nguyen-Deroche Tle, N., Caruso, A., Le, T.T., Bui, T.V., Schoefs, B., Tremblin, G. and Morant-Manceau, A. (2012) Zinc affects differently growth, photosynthesis, antioxidant enzyme activities and phytochelatin synthase expression of four marine diatoms. ScientificWorldJournal, 2012, 982957.

Porra, R.J. (2002) The chequered history of the development and use of simultaneous equations for the accurate determination of chlorophylls a and b. Photosynth. Res., 73, 149-156.

Rivera-Serrano, E.E., Rodriguez-Welsh, M.F., Hicks, G.R. and Rojas-Pierce, M. (2012) A small molecule inhibitor partitions two distinct pathways for trafficking of tonoplast intrinsic proteins in Arabidopsis. PLoS One, 7, e44735.

Robinson, S.P. (1985) Osmotic adjustment by intact isolated chloroplasts in response to osmotic stress and its effect on photosynthesis and chloroplast volume. Plant Physiol., 79, 996-1002.

Schmid, M., Davison, T.S., Henz, S.R., Pape, U.J., Demar, M., Vingron, M., Scholkopf, B., Weigel, D. and Lohmann, J.U. (2005) A gene expression map of Arabidopsis thaliana development. Nat. Genet., 37, 501-506.

Schuldinger, S., Rottenberg, H. and Avron, M. (1972) Determination of $\Delta \mathrm{pH}$ in Chloroplasts. 2.Fluorescent amines as a probe for the determination of $\Delta \mathrm{pH}$ in chloroplasts. Eur. J. Biochem., 25, 64-70. 
Schussler, M.D., Alexandersson, E., Bienert, G.P., Kichey, T., Laursen, K.H., Johanson, U., Kjellbom, P., Schjoerring, J.K. and Jahn, T.P. (2008) The effects of the loss of TIP1;1 and TIP1;2 aquaporins in Arabidopsis thaliana. Plant J., 56, 756-767.

Taira, M., Valtersson, U., Burkhardt, B. and Ludwig, R.A. (2004) Arabidopsis thaliana GLN2-encoded glutamine synthetase is dual targeted to leaf mitochondria and chloroplasts. Plant Cell, 16, 2048-2058.

Thuswaldner, S., Lagerstedt, J.O., Rojas-Stutz, M., Bouhidel, K., Der, C., LeborgneCastel, N., Mishra, A., Marty, F., Schoefs, B., Adamska, I., Persson, B.L. and Spetea, C. (2007) Identification, expression, and functional analyses of a thylakoid ATP/ADP carrier from Arabidopsis. J. Biol. Chem., 282, 8848-8859.

Tomizioli, M., Lazar, C., Brugiere, S., Burger, T., Salvi, D., Gatto, L., Moyet, L., Breckels, L.M., Hesse, A.M., Lilley, K.S., Seigneurin-Berny, D., Finazzi, G., Rolland, N. and Ferro, M. (2014) Deciphering thylakoid sub-compartments using a mass spectrometry-based approach. Mol Cell Proteomics, 13, 2147-2167.

Uehlein, N., Lovisolo, C., Siefritz, F. and Kaldenhoff, R. (2003) The tobacco aquaporin NtAQP1 is a membrane CO2 pore with physiological functions. Nature, 425, 734737.

Uehlein, N., Otto, B., Hanson, D.T., Fischer, M., McDowell, N. and Kaldenhoff, R. (2008) Function of Nicotiana tabacum aquaporins as chloroplast gas pores challenges the concept of membrane CO2 permeability. Plant Cell, 20, 648-657.

Van, T.V., Heinze, T., Buchholz, J. and Rumberg, B. (1987) Quantitative relationship between 9-aminoacridine fluorescence quenching and internal $\mathrm{pH}$ in broken chloroplasts. In Progress in photosynthesis research (J., B. ed. Netherlands: Martinus Nijhoff Publishers pp. III.2.189-III.182.192.

Wang, M., Ding, L., Gao, L., Li, Y., Shen, Q. and Guo, S. (2016) The interaction of aqauporins and mineral nutrients in higher plants. Int. J. Mol. Sci. 17: 1229

Zhang, L., Kato, Y., Otters, S., Vothknecht, U.C. and Sakamoto, W. (2012) Essential role of VIPP1 in chloroplast envelope maintenance in Arabidopsis. Plant Cell, 24, 3695-3707. 
bioRxiv preprint doi: https://doi.org/10.1101/2020.09.18.297978; this version posted September 18, 2020. The copyright holder for this preprint (which was not certified by peer review) is the author/funder. All rights reserved. No reuse allowed without permission.

Azeez Beebo et al.

TIP family aquaporins in chloroplasts

Zybailov, B., Rutschow, H., Friso, G., Rudella, A., Emanuelsson, O., Sun, Q. and van Wijk, K.J. (2008) Sorting signals, N-terminal modifications and abundance of the chloroplast proteome. PLoS One, 3, e1994. 
Table 1. Photosynthetic parameters in Col and tip mutants

\begin{tabular}{|c|c|c|c|c|c|c|}
\hline \multirow[b]{2}{*}{ Genotype } & \multirow[b]{2}{*}{$\begin{array}{c}\mathrm{P}_{700}{ }^{+} \mathrm{t}_{1 / 2}{ }^{\mathrm{a}} \\
(\mathrm{ms})\end{array}$} & \multirow[b]{2}{*}{$F_{\mathrm{v}} / F_{\mathrm{m}}$} & \multirow[b]{2}{*}{$\Phi_{\mathrm{II}}$} & \multicolumn{3}{|c|}{$\mathrm{O}_{2}$ evolution } \\
\hline & & & & \multicolumn{3}{|c|}{$\mu \mathrm{mol} \mathrm{O} \mathrm{mg} \mathrm{Chl}^{-1} \mathrm{~h}^{-1}$} \\
\hline \multirow[t]{2}{*}{ Col } & $6.7 \pm 0.8$ & $0.8 \pm 0.0$ & $0.2 \pm 0.0$ & $423 \pm 66$ & $215 \pm 47$ & $695 \pm 111$ \\
\hline & $100 \pm 11 \%$ & $100 \pm 0 \%$ & $100 \pm 12 \%$ & $100 \pm 16 \%$ & $100 \pm 22 \%$ & $100 \pm 10 \%$ \\
\hline tipl;2 & $90 \pm 8 \%$ & $99.7 \pm 1.2 \%$ & $75 \pm 21 \% *$ & $105 \pm 21 \%$ & $106 \pm 22 \%$ & $103 \pm 22 \%$ \\
\hline tip $2 ; 1$ & $90 \pm 6 \%$ & $99.5 \pm 0.9 \%$ & $75 \pm 16 \% *$ & $106 \pm 18 \%$ & $106 \pm 22 \%$ & $105 \pm 20 \%$ \\
\hline tipl;2/2;1 & $90 \pm 4 \%$ & $99.1 \pm 0.9 \%$ & $79 \pm 8 \% *$ & $103 \pm 14 \%$ & $105 \pm 19 \%$ & $101 \pm 18 \%$ \\
\hline
\end{tabular}

Plants were grown for 6 weeks and dark-adapted overnight before recording the halftime of $\mathrm{P}_{700}{ }^{+}\left(\mathrm{t}_{1 / 2}\right)$ and $F_{\mathrm{V}} / F_{\mathrm{m}}$ in intact leaves. $\Phi_{\mathrm{II}}$ was recorded in intact leaves following illumination. Steady-state rate of PSIImediated $\mathrm{O}_{2}$ evolution was measured in isolated thylakoids using dimethyl-benzoquinone as artificial electron acceptor in the presence of the $\mathrm{H}^{+}$uncoupler nigericin. Linear electron transfer rate (ETR) was measured using methyl viologen and sodium azide under similar conditions. The $\mathrm{Cytb}_{6} \mathrm{f}$ activity was determined using reduced duroquinol) as electron donor and methyl viologen as PSI acceptor. Absolute values are given for Col, whereas mutant data are expressed relative to Col. All values are means \pm SD $(n=5-10)$. Significantly different values within the same column are indicated with asterisk (Student's t-test, $P<0.05$ ). 


\section{Figure Legends}

Figure 1. TIPs localization in Arabidopsis chloroplasts.

(a) Representative Western blots with an anti-GFP antibody of leaf protein extracts and chloroplasts purified from plants expressing TIP-GFP fusion proteins and non-transformed plants (Col). The lack of vacuoles in the analyzed preparations was confirmed using anti-VATPase antibodies.

(b) Confocal microscopic images of chloroplasts purified from transgenic (TIPG) and Col plants. Scale bar $=10 \mu \mathrm{m}$. The fluorescent signal had a punctate distribution in chloroplasts. "GFP" represents GFP in the chloroplasts from transformed plants. "Merge" shows the overlap of the GFP and the Chl autofluorescence $(C H L)$, which was used as a marker for chloroplasts.

(c) Representative Western blots with anti-TIP antibodies of leaf extracts, chloroplasts, thylakoids and envelope. The purity of the analyzed preparations was confirmed using antiV-ATPase, $\mathrm{H}^{+}$-ATPase, Tic40 and Lhcb2 antibodies.

Figure 2. Molecular and growth characteristics of the tip mutants.

(a) Structure of the Arabidopsis TIP1;2 and TIP2;1 genes is shown, indicating the position of the transposon insert (dSpm).

(b) Quantitative RT-PCR shows level $<3 \%$ for TIP transcripts in the corresponding genotype.

(c) Western blots using a common anti-TIP1;1/TIP1;2 and anti-TIP2;1 antibodies of leaf protein extracts confirmed the lack of the corresponding TIP protein in the respective mutants.

(d) Representative photos at 8 weeks under $120 \mu \mathrm{mol}$ photons $\mathrm{m}^{-2} \mathrm{~s}^{-1}$.

(e) Chlorophyll (Chl) content index at an age of 8 weeks.

(f) Shoot fresh and dry weight as a function of plant age. The plotted data in (e) and (f) are means \pm SD $(n=10-15)$. No significant differences were observed between the mutants and Col (Student's t-test, $P>0.05$ ).

Figure 3. Changes in chloroplast morphology upon hypo- and hyper-osmotic treatments. 
Light microscopic images were taken on Percoll gradient chloroplasts following a 5 min incubation in $0.3 \mathrm{M}$ sucrose buffer (control), in water, $0.5 \mathrm{M} \mathrm{NaCl}$ or $1 \mathrm{M}$ mannitol. Scale bar $=5 \mu \mathrm{m}$. The Col and tip2;1 mutant chloroplasts undergo considerable morphological changes, whereas the tipl;2 and tip1;2/tip2;1 mutant chloroplasts appear to be resistant to the osmotic treatments.

Figure 4. Changes in $90^{\circ}$ light scattering in thylakoids during illumination and osmotic treatment.

Isolated thylakoids were either illuminated at $1,000 \mu \mathrm{mol}$ photon $\mathrm{m}^{-2} \mathrm{~s}^{-1}$ in the presence of methyl viologen or incubated in darkness in a buffer containing a series of sorbitol concentrations (for buffer details, see Experimental Procedures). As control, Col thylakoids were incubated in a buffer where the sorbitol was replaced with corresponding volumes of water. Thylakoid lumen volume changes were measured as light scattering change at 550 $\mathrm{nm}\left(\Delta \mathrm{A}_{550}\right)$. The plotted values are means $\pm \mathrm{SD}(\mathrm{n}=5-7)$. Insert: Formation and relaxation rate constants of the light-induced scattering change.

Figure 5. Photosynthetic activity and light sensitivity.

(a) left: Light response curves of $\mathrm{O}_{2}$ evolution rates recorded in leaf discs $(\mathrm{n}=3-5)$. Right: Maximal $\mathrm{O}_{2}$ evolution from fitted light response curves.

(b) Light response curves of electron transfer rates $(E T R)$ recorded on intact plants $(n=10)$.

(c) Net $\mathrm{CO}_{2}$ assimilation rates $\left(A_{n}\right)$ versus intercellular $\mathrm{CO}_{2}$ concentrations $\left(C_{i}\right)$ recorded in leaf discs using light of $1,800 \mu \mathrm{mol}$ photon $\mathrm{m}^{-2} \mathrm{~s}^{-1}(\mathrm{n}=3-4)$.

(d) $F_{\mathrm{v}} / F_{\mathrm{m}}$ of intact plants as a function of number of days in high light conditions (800 $\mu \mathrm{mol}$ photon $\left.\mathrm{m}^{-2} \mathrm{~s}^{-1}\right)(\mathrm{n}=5)$.

(e) Slow kinetics for induction of non-photochemical quenching (NPQ) formation recorded on intact plants using light of $800 \mu \mathrm{mol}$ photon $\mathrm{m}^{-2} \mathrm{~s}^{-1}(\mathrm{n}=3-4)$.

(f) Electrochromic shift $\left(\right.$ ECS $\left._{\text {total }}\right)$ recorded on intact plants illuminated at $500 \mu$ mol photon $\mathrm{m}^{-2} \mathrm{~s}^{-1}(\mathrm{n}=5-7)$.

(g) $\Delta \mathrm{pH}$ recorded as fluorescence of 9-aminoacridine in isolated thylakoids $(\mathrm{n}=7-10)$. 
bioRxiv preprint doi: https://doi.org/10.1101/2020.09.18.297978; this version posted September 18, 2020. The copyright holder for this preprint (which was not certified by peer review) is the author/funder. All rights reserved. No reuse allowed without permission.

Azeez Beebo et al.

TIP family aquaporins in chloroplasts

The plotted values are means \pm SD (a-g). Significantly different values for parameters in the mutants relative to $\mathrm{Col}$ are indicated with asterisk (Student's t-test, $P<0.05$ ). 
Control

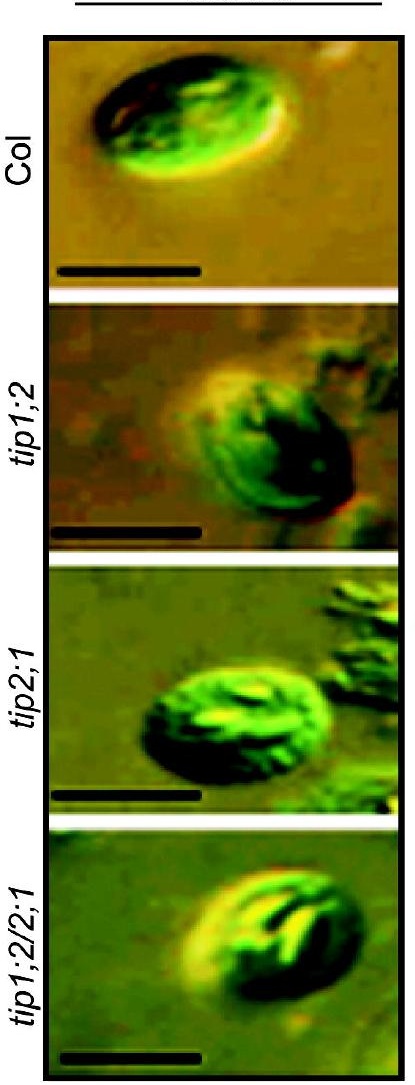

Water
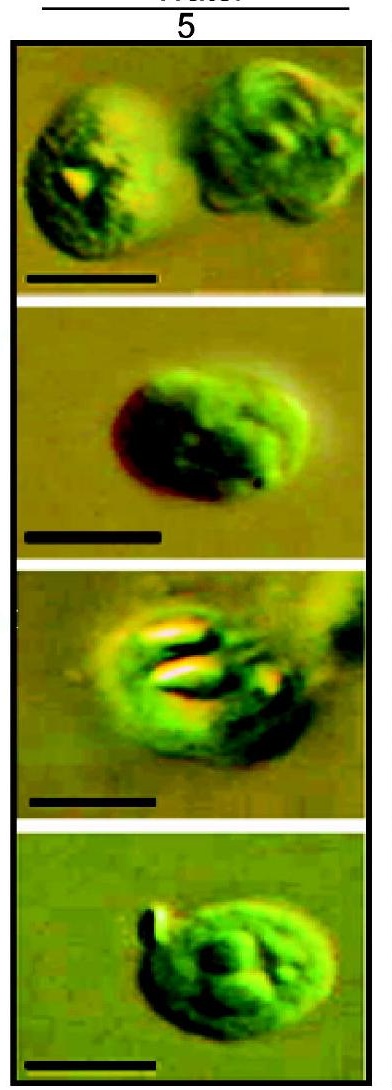

$\mathrm{NaCl}(0.5 \mathrm{M})$
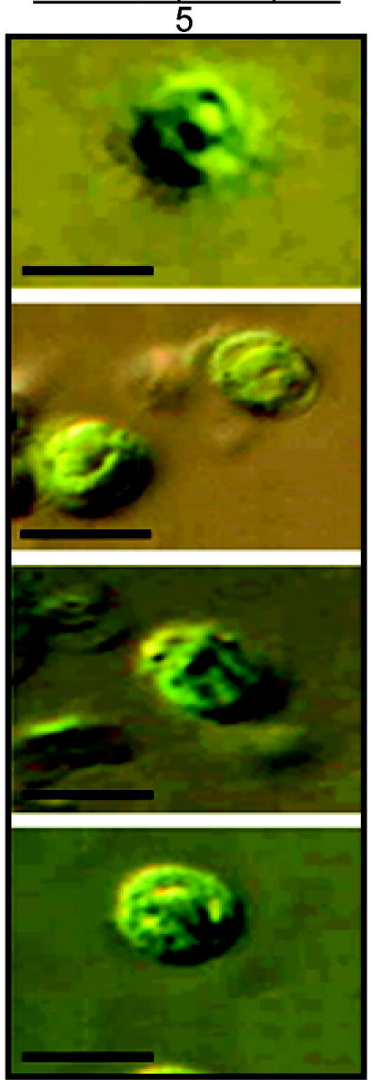

Mannitol (1 M) 5

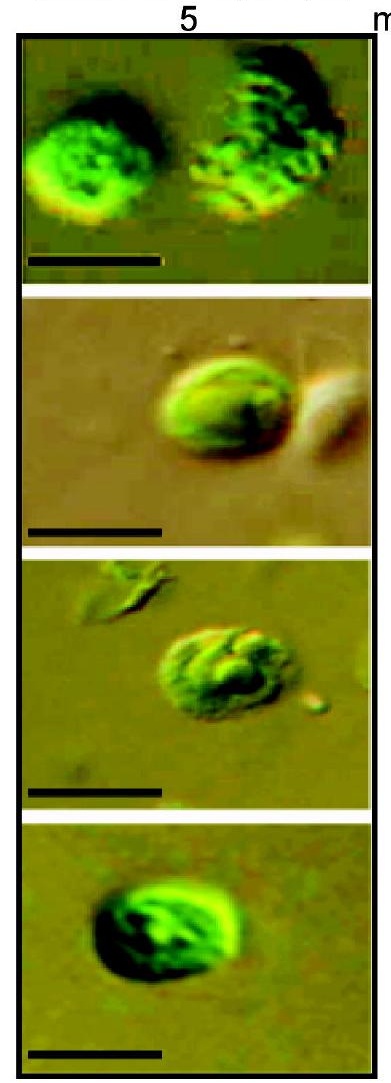




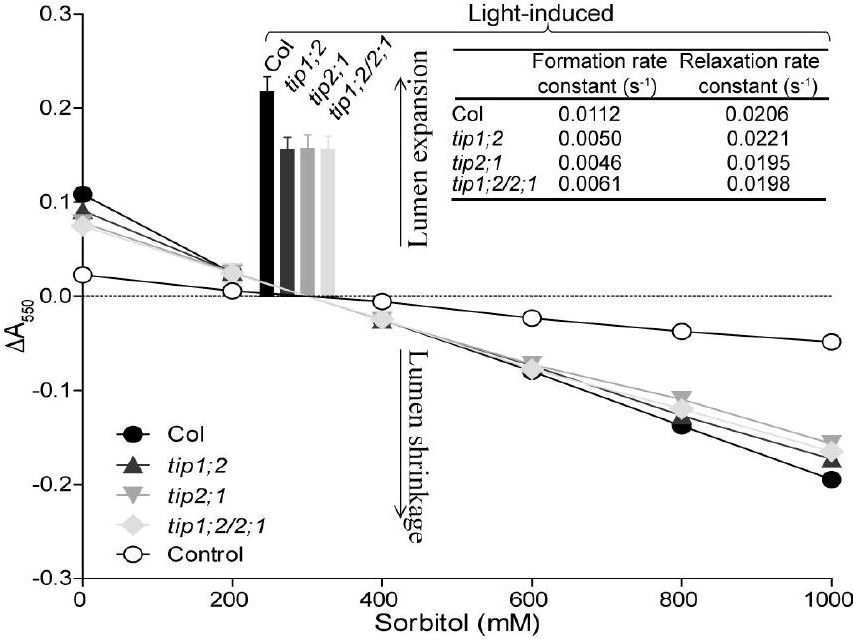



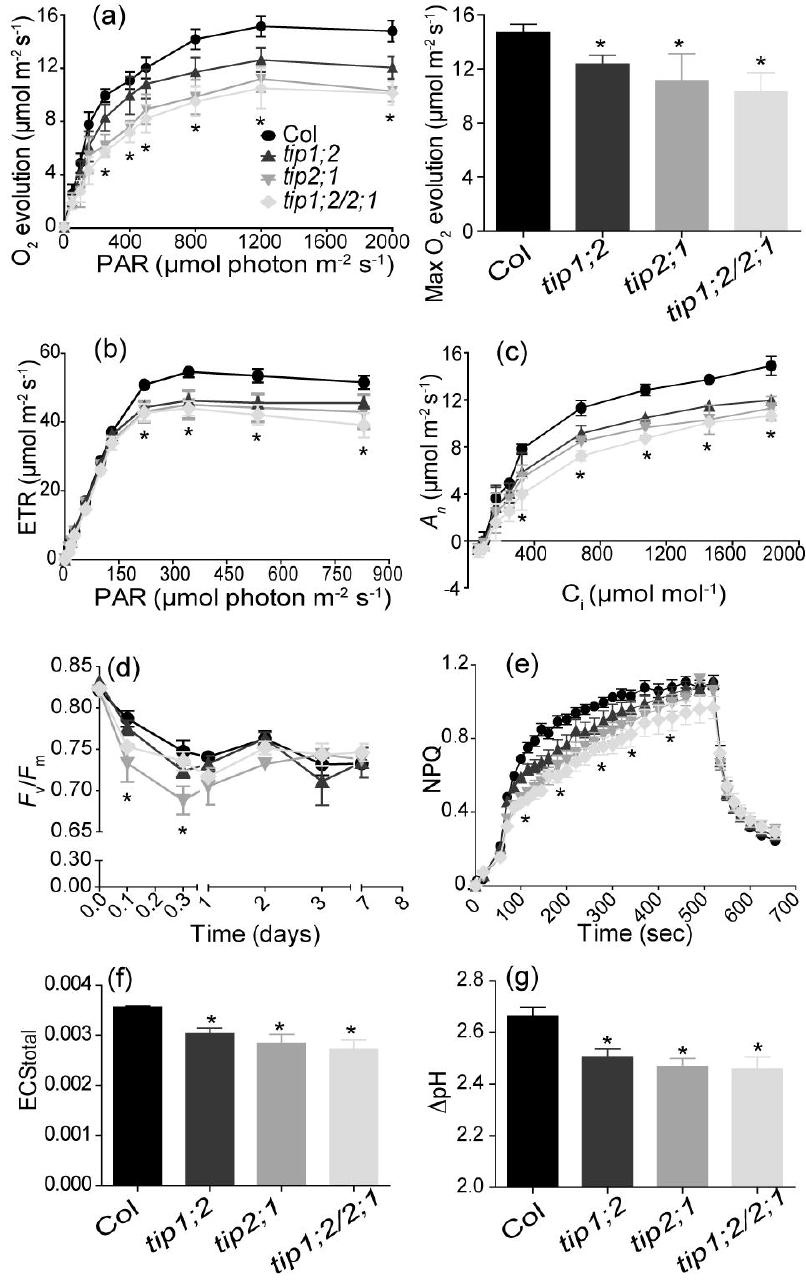